\title{
Deterministic tsunami hazard map for India
}

\author{
J. Dhanya* and S. T. G. Raghukanth \\ Department of Civil Engineering, Indian Institute of Technology Madras, Chennai 600 036, India
}

\begin{abstract}
The present work aims to ascertain the deterministic tsunami hazard map for maximum wave height along the Indian coastline due to subduction events in the Sumatra region. The region between $15^{\circ} \mathrm{S}-30^{\circ} \mathrm{N}$ lat. and $50^{\circ} \mathrm{E}-115^{\circ} \mathrm{N}$ long. was modelled in using Geoclaw, which discretizes and solves the shallow-water wave equation using adaptive finite volume algorithm. The developed model was suitably validated for the available tidal gauge and altimeter data for the $2004 M_{\mathrm{w}}$ 9.12 Sumatra earthquake with input source characteristics from the SRCMOD data. Further, a sensitivity study based on slip variability and location was conducted which revealed that near-field stations were highly sensitive at all locations while far-field stations were more sensitive towards source locations. Furthermore, 25 non-Gaussian slip fields were generated for the maximum possible event $\left(M_{\mathrm{w}}\right.$ 9.12) for the region and placed suitably along the active Sumatra subduction region. Then the wave heights from all the simulations were assembled to determine the deterministic tsunami hazard values with respect to maximum wave heights for the coastal regions of India and adjoining regions. The results will find application in the design of structures along the coastline of the study region.
\end{abstract}

Keywords: Tsunami, Sumatra Region, India, Hazard, Design of coastal structures.

ONE of the most disastrous consequences of an earthquake in the ocean floor is the triggered tsunami. Hence, an estimate of the hazard due to tsunami is essential for the design of structures along the coastal regions of tsunami-prone areas. One of the most active and tsunamigenic zones that has significant influence on the coastline of India is the Sumatra subduction region. The catastrophe caused by the $M_{\mathrm{w}} 9.12$ Sumatra earthquake is a clear example of the socio-economic importance of such hazard preparedness. Furthermore, owing to the highly nonlinear behaviour of the generated tsunami waves, formulation of ground motion prediction equations as practised for seismic hazard estimations is not feasible in this case. Most of the preliminary approaches to estimate tsunami hazard used statistical analysis on historic data ${ }^{1-3}$. The first probabilistic tsunami hazard estimate based on synthetic data was done by Houston and Garcia ${ }^{4}$. Rikitake and $\mathrm{Aida}^{5}$ combined the numerically generated tsunami waves and probability of earthquake occurrence to eva-

*For correspondence. (e-mail: dhanyaj17@gmail.com) luate the possible tsunami at a site. Similarly, Geist and Parsons ${ }^{6}$ and González et al. ${ }^{7}$ proposed methodologies to estimate hazard due to wave height and maximum extent of inundation. Lorito et $a l^{8}{ }^{8}$ derived the seismic probability tsunami hazard algorithm using event tree and hierarchical cluster analysis. However, simulation of synthetic waves generated for all possible scenarios for seismogenic zones is challenging and computationally expensive. Hence a conservative approach might be to resort to simulations with the worst possible scenario along the potential region. Such analysis is important for the Sumatra subduction zone and the areas influenced by it. Figure 1 shows the active tectonics of the region from seismicity distribution. In the last 250 years, there were around seven great earthquakes $\left(M_{\mathrm{w}}>8\right)$ in this region. These are the 1797 $M_{\mathrm{w}} 8.7,1833 M_{\mathrm{w}} 9,1861 M_{\mathrm{w}} 8.5,2004 M_{\mathrm{w}} 9.12,2005 M_{\mathrm{w}}$ 8.6, $2007 M_{\mathrm{w}} 8.4$ and $2012 M_{\mathrm{w}} 8.6$ events. Bilham et al. ${ }^{9}$ gave an account of events in $1847,1881 M_{\mathrm{w}} 7.9$ and 1941 $M_{\mathrm{w}} 7.7$, other than the $2004 M_{\mathrm{w}} 9.12$ Sumatra earthquake which were large enough to cause tsunami-like waves in the Indian Ocean. Additionally, from the recurrence characteristics, the region has the possibility of largemagnitude earthquakes that can trigger tsunami waves with a return period of $114-200$ years ${ }^{10,11}$. In this respect, for the coastline along India, various attempts have been made to arrive at the probable tsunami hazard and resultant vulnerability ${ }^{12-14}$. However, the estimates are based on only idealized source segments with uniform slip. Whereas several studies have pointed to the considerable influence of earthquake source complexities on the ground motion and the subsequent tsunami waves ${ }^{15-17}$. Thus, in the case of large events, accounting for the source variabilities and rupture propagation characteristics is essential. Dhanya and Raghukanth ${ }^{18}$ proposed a finite element model for the Sumatra region to simulate reliable earthquake ground motions. However, ground motion simulations using the proposed model are computationally expensive. Recently, Dhanya and Raghukanth ${ }^{19}$ have conducted a study to understand the effects of different source models reported for the $2004 M_{\mathrm{w}} 9.12$ Sumatra earthquake on tsunami wave propagation. However, they have not performed a detailed hazard characterization of the region by considering the potential rupture zone that affects the coastal regions of India.

The present study aims to develop a deterministic tsunami hazard map for maximum wave height along the coastal regions of India based on simulations accounting for source variabilities. First, a simple algorithm for 


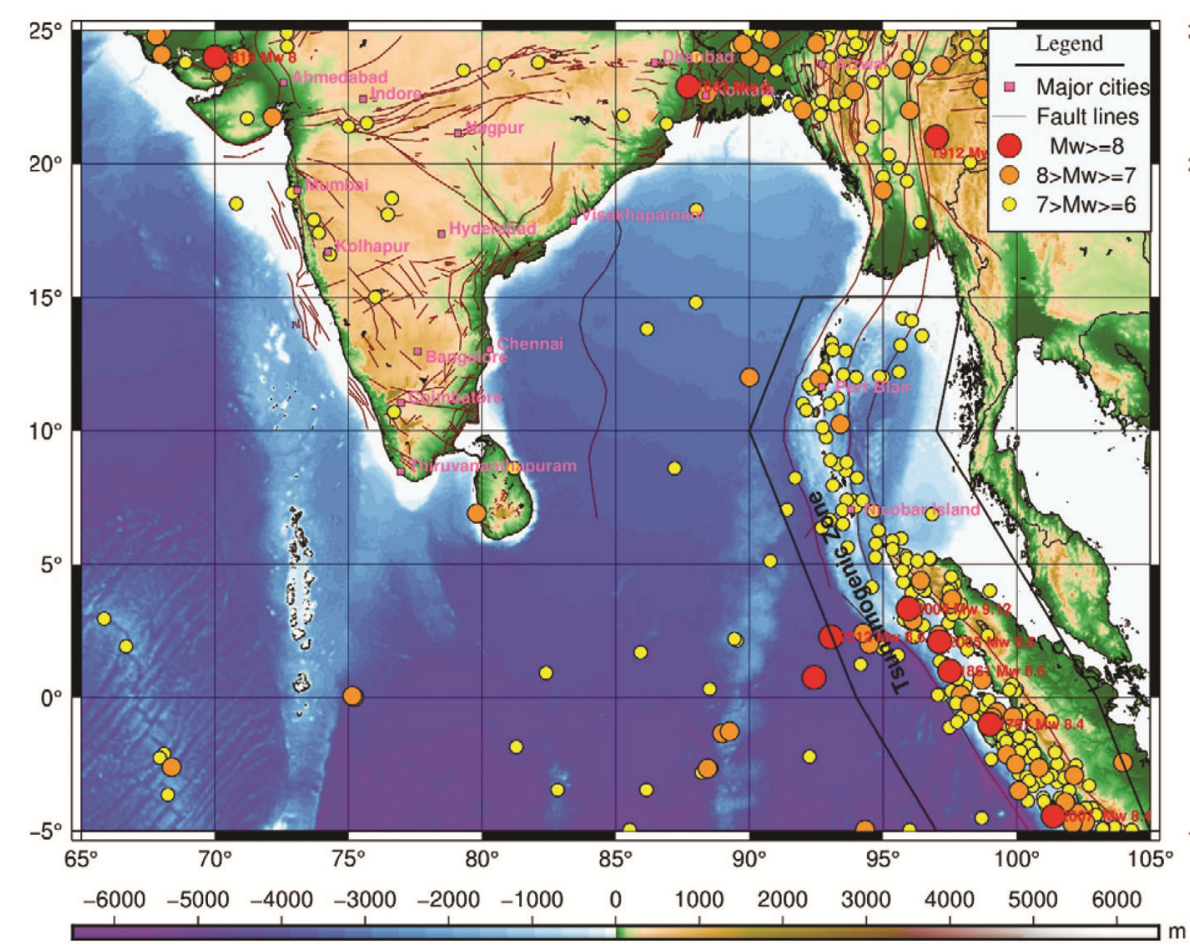

Figure 1. Tectonic setting of the study region along with topography (bathymetry). The tsunamigenic zone of the region is marked with bold black lines.

dynamic ground motion using quasi-static Okada's solutions is tested for the region. Then, the same is implemented in the generation of tsunami waves based on adaptive finite volume methodology proposed by George ${ }^{20}$, using Geoclaw. Further, possible synthetic slip models are derived based on the non-Gaussian random field algorithm proposed by Dhanya and Raghukanth ${ }^{21}$. The sensitivity of tsunami waves towards slip variability and location is tested. Furthermore, the potential rupture models for the $M_{\mathrm{w}} 9.12$ scenario event is suitably applied along the subduction region at Sumatra to arrive at the deterministic tsunami hazard corresponding of maximum wave height along the coastline of the Bay of Bengal. The results are important in the design of structures along the coast.

\section{Seismically induced tsunami wave generation}

In this work, a simplified model has been adopted to simulate ground motions for earthquakes in the Sumatra region. Then, a well-tested tsunami generation algorithm is implemented and validated for the region. A brief description on the methodologies adopted for ground motion simulation and subsequent tsunami wave generation is given below.

\section{Ground displacements}

The spectral finite element model developed for the South Asian region and corresponding simulations reported by Dhanya and Raghukanth ${ }^{18}$ pointed to the sen- sitivity of slip distribution on ground displacements. However, the method proposed by them ${ }^{18}$ is computationally expensive as it takes almost three days using 256 processors to complete one ground motion simulation. Hence, application of the corresponding model is computationally inefficient to simulate of a suite of scenario events. Moreover, tsunami waves are typically longperiod waves and are hence sensitive towards the lowfrequency characteristics of ground motion. Considering this aspect, the input ground motions were simulated following Dutykh et al. ${ }^{22}$, where a quasi-static approach has been proposed for the Okada's ${ }^{23}$ solution. Okada's solution for the displacement field $s_{i}\left(x_{1}, x_{2}, x_{3}\right)$ due to a dislocation $\delta s_{j}\left(\xi_{1}, \xi_{2}, \xi_{3}\right)$ in isotropic elastic half space across a surface $(\Sigma)$ can be expressed as

$$
s_{i}=\frac{1}{F} \iint_{\Sigma} \delta s_{j}\left[\lambda \delta_{i j} \frac{\partial s_{i}^{n}}{\partial \xi_{n}}+\mu\left(\frac{\partial s_{i}^{j}}{\partial \xi_{k}}+\frac{\partial s_{i}^{k}}{\partial \xi_{j}}\right)\right] v_{k} d \sum,
$$

where $\lambda$ and $\mu$ are the Lame constants, $v_{k}$ is the included angle cosine of the normal to the surface element $d \Sigma$ and $s_{i}^{j}$ is the displacement component $i$ due to force $F$ at point $\left(\xi_{1}, \xi_{2}, \xi_{3}\right)$ in the $j$ th direction. Equation (1) can be used to analyse the static displacement field as well as the strain changes caused by an earthquake. The detailed expression for $s_{i}$ along each direction with respect to the faulting mechanism is given in ref. 23. It should be noted that eq. (1) can be applied for a finite fault by integrating along length and width. The method is widely used in 

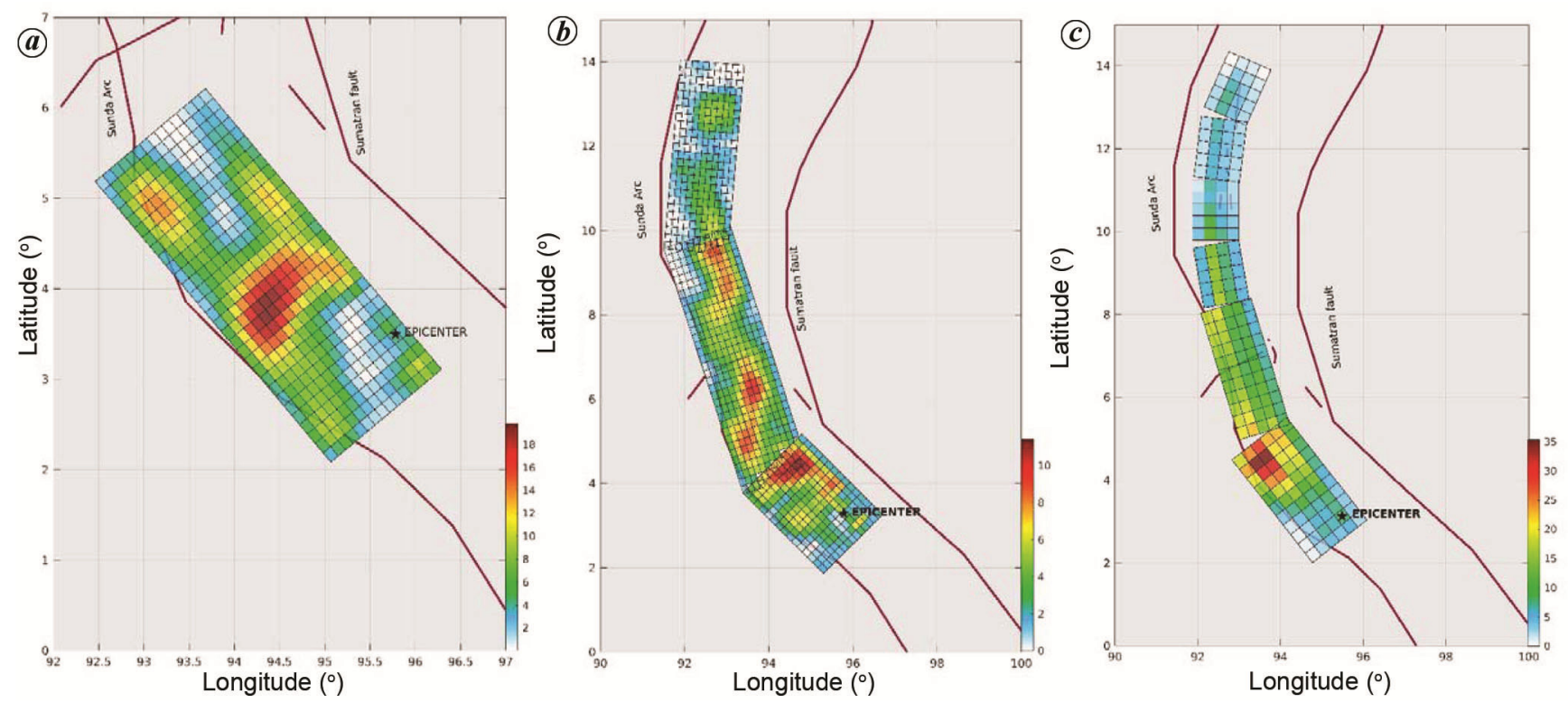

Figure 2. Slip distribution (m) for the $2004 M_{w} 9.12$ Sumatra earthquake available in the SRCMOD database. $\boldsymbol{a}$, Ji ${ }^{25}$; $\boldsymbol{b}$, Ammon et al. ${ }^{26}$; c, Rhie et al. ${ }^{27}$.

ground displacement simulations. For larger slips, first the rupture plane is discretized into finite segments called sub-faults. Here, for brevity the displacement field at the surface due to the $i$ th sub-fault from Okada's solution is symbolized as $s_{i}\left(\lambda, \mu, \delta, \phi, d_{i}\right)$, where $\delta$ is the dip angle, $\phi$ rake angle and $d_{i}$ corresponds to slip value at the $i$ th subfault. Further, the dynamic solution for the displacement field is obtained by assuming a trigonometric function. The resultant rate of evolution can be expressed as

$$
f(t)=\mathcal{H}\left(t-t_{\mathrm{r}}\right)+\frac{1}{2} \mathcal{H}(t) \mathcal{H}\left(t_{\mathrm{r}}-t\right)\left(1-\cos \left(\pi t / t_{\mathrm{r}}\right)\right)
$$

where $t_{\mathrm{r}}$ is the rise time and $\mathcal{H}$ is the Heaviside step function. Here, $f(t)$ is zero when $t<0$ and 1 when $t \geq t_{\mathrm{r}}$. The complete evolution of the displacement field is estimated by suitably integrating the contribution from each subfault along with its rupture initiation time $\left(t_{i}\right)$. This corresponding displacement field at each instant $(S(t))$ can be expressed as

$$
S(t)=\sum_{i=1}^{N x \times N z} \mathcal{H}\left(t-t_{i}\right) f\left(t-t_{i}\right) s_{i}\left(\left(\lambda, \mu, \delta, \phi, d_{i}\right)\right),
$$

where $N x, N z$ are the number of sub-faults along the strike and dip directions respectively. The relation of Somerville et $a l^{24}$ was utilized to estimate the rise time $t_{r}$ as

$$
t_{\mathrm{r}}=2.303 \times 10^{-9} M_{0}^{1 / 3},
$$

where $M_{0}=\mu \times \mathrm{d} x \times \mathrm{d} y \times d$ is the seismic moment of each sub-fault having dimensions $[\mathrm{d} x ; \mathrm{d} y]$, slip value $d_{\mathrm{s}}$ and rupture modulus $\mu$. The performance of the model can be evaluated by a comparison with the results in ref.
18 for the $2004 M_{w} 9.12$ Sumatra earthquake. Here, slip models for the 2004 Sumatra event proposed by several researchers ${ }^{25-27}$ available at http://equake-rc.info/srcmod/ (SRCMOD database) have been used for comparison. Figure 2 shows the corresponding slip field.

The dynamic displacement field of the $2004 M_{w} 9.12$ Sumatra earthquake was estimated, using the quasi-static approach, by substituting the rupture characteristics as reported for the slip fields in the SRCMOD database. The time step $(\delta t)$ for the simulations was decided considering the slip discretization and total rupture duration. The simulated displacement time histories were first compared with those reported in ref. 18. It should be noted that the quasi-static Okada's solution requires only $10 \mathrm{~min}$ using a single processor to generate a time history of length 33 min. The quasi-static Okada's solution captures well the predominant feature of displacement time histories. Figure 3 shows the GRD obtained from the quasi-static Okada model. Here the displacements were obtained for a region between $90^{\circ}$ and $100^{\circ} \mathrm{E}$ long. and $0^{\circ}$ and $15^{\circ} \mathrm{N}$ lat. with a resolution of $0.017^{\circ} \times 0.017^{\circ}$. Comparing the simulated ground residual displacement figure with that in ref. 18 , it can be noted that both models render similar amplitudes and spatial patterns. From the comparison, it is observed that the tsunami simulations can be performed with the ground displacement from computationally efficient quasi-static Okada's solution. A suitable methodology for the generation of synthetic tsunami waves is discussed further.

\section{Tsunami wave simulations}

The ground displacement explained in the previous section is suitably used to simulate tsunami waves. The 

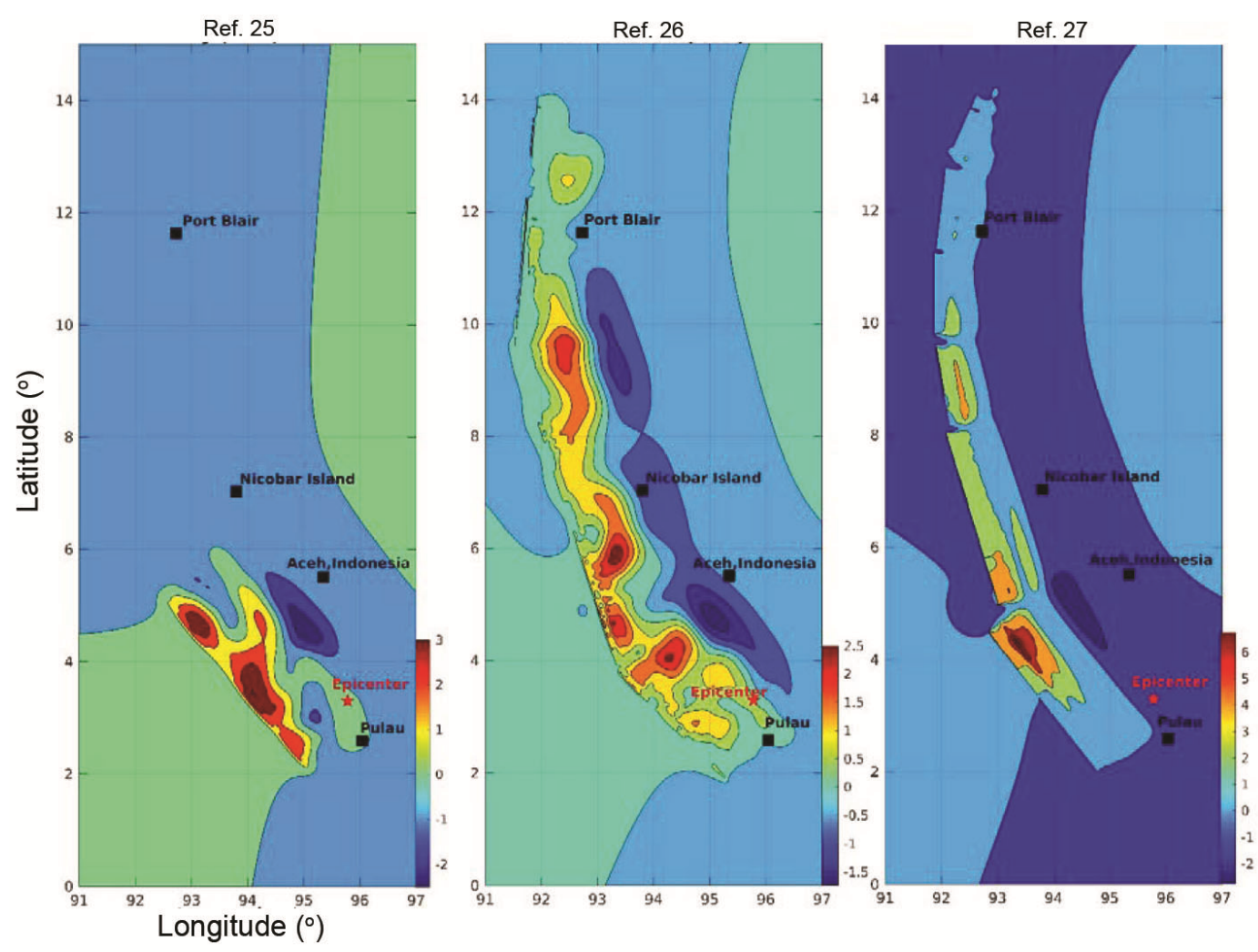

Figure 3. Ground residual displacement (GRD) contours (m) for vertical direction corresponding to the $2004 M_{w} 9.12$ Sumatra earthquake slip models reported in the SRCMOD database simulated using quasi-static Okada solutions.

tsunami wave generation and propagation are highly nonlinear and complicated due to the non-homogeneous boundary conditions. Hence, framing a closed-form analytical solution is impossible to derive, and does not exist due to bathymetry and other nonlinearities. Therefore, the typical practice is to resort to numerical techniques. The advancement in the computational domain aided in handling large data and complex equations effectively with less computation time. Typically, tsunami waves are modelled using a shallow water wave equation (SWE) which is derived from the depth averaging of the NavierStokes equation ${ }^{28}$. The corresponding equation can be expressed as

$$
\frac{\partial q}{\partial t}+\frac{\partial f(q)}{\partial x}+\frac{\partial g(q)}{\partial y}=\Psi(q, x, y)
$$

where $q$ represents the vector of unknowns, $f(q)$ and $g(q)$ is the vector of corresponding fluxes and $\Psi(q, x, y)$ is a vector of representing the source terms, such that

$$
q=\left[\begin{array}{c}
h \\
h \dot{u}_{x} \\
h \dot{u}_{y}
\end{array}\right], f(q)=\left[\begin{array}{c}
h \dot{u}_{x} \\
h \dot{u}_{x}^{2}+\frac{1}{2} g h^{2} \\
h \dot{u}_{x} \dot{u}_{y}
\end{array}\right]
$$

$$
g(q)=\left[\begin{array}{c}
h \dot{u}_{y} \\
h \dot{u}_{x} \dot{u}_{y} \\
\frac{1}{2} g h^{2}+h \dot{u}_{y}{ }^{2}
\end{array}\right], \Psi=\left[\begin{array}{c}
0 \\
-g h \frac{\partial B}{\partial x} \\
-g h \frac{\partial B}{\partial y}
\end{array}\right],
$$

where $h(x, y, t)$ is the fluid depth, $g$ the gravitational constant, $B(x, y)$ the bathymetry or elevation of the ocean bed, $\dot{u}_{x}$ and $\dot{u}_{y}$ are the fluid velocity in the $x$ and $y$ direction respectively. This belongs to the class of hyperbolic equation as we can obtain real and distinct eigenvalues for the Jacobian matrix $f^{\prime}(q)$ and $g^{\prime}(q)$. Here, the eigenvalues represent the velocity of propagation of the wave. The water surface elevation $\mathbf{H}(\mathbf{x}, \mathbf{y}, \mathbf{t})$ is given by

$$
\mathbf{H}(\mathbf{x}, \mathbf{y}, \mathbf{t})=h(x, y, t)+B(x, y) .
$$

There are various numerical schemes to solve eq. (5). Some of the earlier studies used the Taylor series expansion-based finite difference schemes. Later, more scientific and sophisticated numerical schemes were used to estimate real-time global-scale tsunami simulation and run-ups. A few among them are method of splitting tsunami $(\mathrm{MOST})^{29}$, Imamura models ${ }^{30,31}$, etc. based on finite difference method (FDM). However, FDM techniques have a limitation as the solution becomes unstable and 
unrealistic with complicated geometry at steep gradients having unstructured elements. A suitable approach to circumvent these limitations is finite element method (FEM) and finite volume method (FVM) formulations, where the integral form or weak form of the partial differential equation is evaluated. Though the accuracy of the estimates increases using FEM, the method might be nonconservative leading to spurious outputs, especially at discontinuities. Whereas we observe that FVM preserves conservativeness in its discretization. Thus, the integral form of SWE can be written as

$$
\begin{aligned}
& \frac{\partial}{\partial t} \int_{y_{1}}^{y_{2}} \int_{x_{1}}^{x_{2}} q(x, y, t) \mathrm{d} x \mathrm{~d} y+\int_{y_{1}}^{y_{2}}(f(q))_{x_{1}}^{x_{2}} \mathrm{~d} y \\
& \quad+\int_{x_{1}}^{x_{2}}(g(q))_{y_{1}}^{y_{2}} \mathrm{~d} x=-\int_{y_{1}}^{y_{2}} \int_{x_{1}}^{x_{2}} \Psi q(x, y, t) \mathrm{d} x \mathrm{~d} y .
\end{aligned}
$$

The initial condition for tsunami wave propagation is the displacement field $(S)$ of the sea level due to fault rupture such that

$$
\mathbf{H}\left(x, y, t_{0}\right)=S\left(x, y, t_{o}\right) .
$$

The other boundary conditions are the bathymetry $(B)$ which is taken equivalent to the digital elevation map (DEM) available for the region. Furthermore, friction of the ground needs to be accounted in inundation modelling. This friction is taken care of by adding some additional friction drag coefficients in the momentum in $\Psi$ along with $B$ like $-D_{r}\left(h, \dot{u}_{x}, \dot{u}_{y}\right) h \dot{u}_{x}$ and $-D_{r}\left(h, \dot{u}_{x}, \dot{u}_{y}\right) h \dot{u}_{y}$ such that

$$
D_{r}=n^{2} g h^{(2 / 3)} \sqrt{\dot{u}_{x}^{2}+\dot{u}_{y}^{2}},
$$

where $n$ is the Manning's constant typically taken as 0.025 and $g$ is the gravitational constant.

For facilitating numerical integration, the computational domain needs to be discretized into $\Delta x \times \Delta y$. For each cell $C_{i j}$, a constant function $Q$ is assumed for a particular time step $k$, given by

$$
Q_{i j}^{k} \approx \frac{1}{V_{i j}} \int_{C_{i j}} q\left(x, y, t_{k}\right) \mathrm{d} x \mathrm{~d} y .
$$

The second-order accurate discretized form of the expression can be written as

$$
\begin{aligned}
Q_{i j}^{k+1} & =Q_{i j}^{k}-\frac{\Delta t}{\Delta x}\left(A^{+} \Delta Q_{i-1 / 2, j}+A^{-} \Delta Q_{i+1 / 2, j}\right) \\
& -\frac{\Delta t}{\Delta y}\left(B^{+} \Delta Q_{i, j-1 / 2}+B^{-} \Delta Q_{i, j+1 / 2}\right)
\end{aligned}
$$

$$
-\frac{\Delta t}{\Delta x}\left(\tilde{F}_{i+1 / 2, j}-\tilde{F}_{i-1 / 2, j}\right)-\frac{\Delta t}{\Delta y}\left(\tilde{G}_{i, j+1 / 2}-\tilde{G}_{i, j-1 / 2}\right),
$$

where $A^{ \pm} \Delta Q_{i \pm 1 / 2, j}$ is the net flux moving from left to right and $B^{ \pm} \Delta Q_{i, j \pm 1 / 2}$ represents the flux through the other two cell interfaces. $\tilde{F}_{i \pm 1 / 2, j}$ and $\tilde{G}_{i, j \pm 1 / 2}$ are the secondorder correction terms. George ${ }^{20}$ has given a detailed explanation on the expressions of this scheme using Godunov-based Reimann method. In order to account for the stability of the above scheme, Courant stability condition is enforced, whereby the time-step of simulation is chosen such that

$$
\frac{\Delta t}{\Delta x}|a|_{\max }<0.75
$$

where $|a|_{\max }$ represents the maximum speed of the wave, $\Delta t$ is the time step of the simulation and $\Delta x$ is the space step from the special discretization of the domain. The described finite volume scheme has been popularly employed for seismically triggered tsunami modelling ${ }^{32}$. The corresponding methodology also finds application in performing dam-break analysis ${ }^{33}$. Isvoranu and Badescu ${ }^{34}$ utilized the code to perform a parametric study on the possible hydrodynamic behaviour of the Black Sea due to an asteroid impact. Recently, Turmel et al. ${ }^{35}$ used this finite volume scheme to estimate the tsunami wave characteristics for submarine landslides in the north shore of St Lawrence Estuary. The modelling technique is observed to adaptively handle complicated boundary conditions that control the flow regime of fluids. Hence the scheme will be ideal to perform tsunami simulations for hazard estimation.

Thus, the explained tsunami simulation methodology is adopted in the present study to model the region of interest and simulate tsunami waves. The domain characteristics are chosen similar to those considered by Dhanya and Raghukanth ${ }^{19}$. Accordingly, the region between $50^{\circ}-$ $115^{\circ} \mathrm{E}$ long. and $15^{\circ}-30^{\circ} \mathrm{N}$ lat. is considered in the model. The main boundary condition in the model that affects the velocity of propagation of the wave is the bathymetry/ocean bottom elevation. For the present model, we have combined the $1 \mathrm{~min}$ resolution data along with $1 \mathrm{~km}$ resolution data at the shore provided by the National Oceanic and Atmospheric Administration (NOAA), USA (https://www.ngdc.noaa.gov/mgg/bathymetry/relief.html). The finite volume discretization of the region is performed with adaptive mesh refinement. The coarsest grid resolution is $1^{\circ} \times 1^{\circ}$, with four layers of refinement with each layer refined in the ratio 6,4 and 2 . Thus, finest mesh will be of size $0.02^{\circ} \times 0.02^{\circ}$. The initial conditions for simulation are the dynamic ground displacement obtained by the procedure explained earlier and zero-velocity field at the ocean surface. The boundary of elements is modelled as non-reflective outflow. The simulations are 

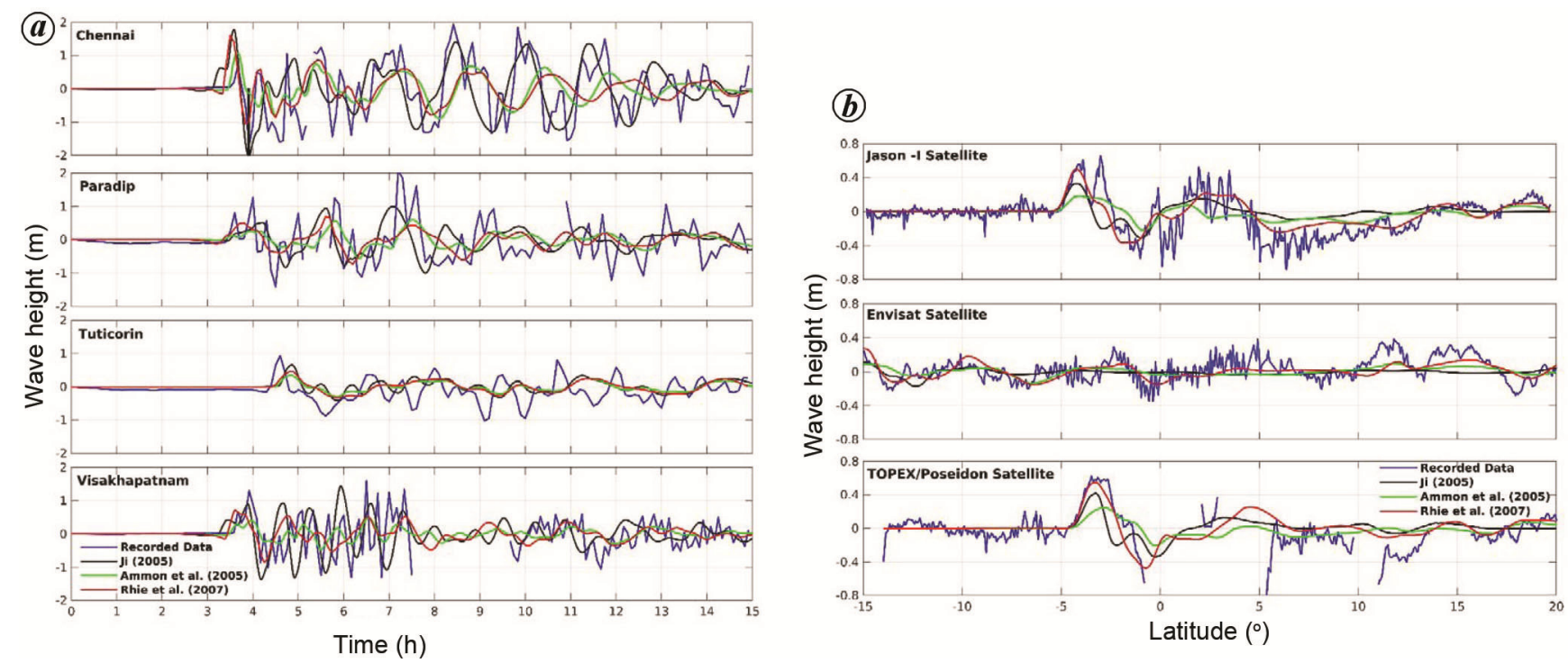

Figure 4. Comparison of tsunami waves simulated using the source characteristics corresponding to slip fields available for the $2004 M_{w} 9.12$ Sumatra earthquake in the SRCMOD database. $\boldsymbol{a}$, Tidal gauge data; $\boldsymbol{b}$, altimeter data.
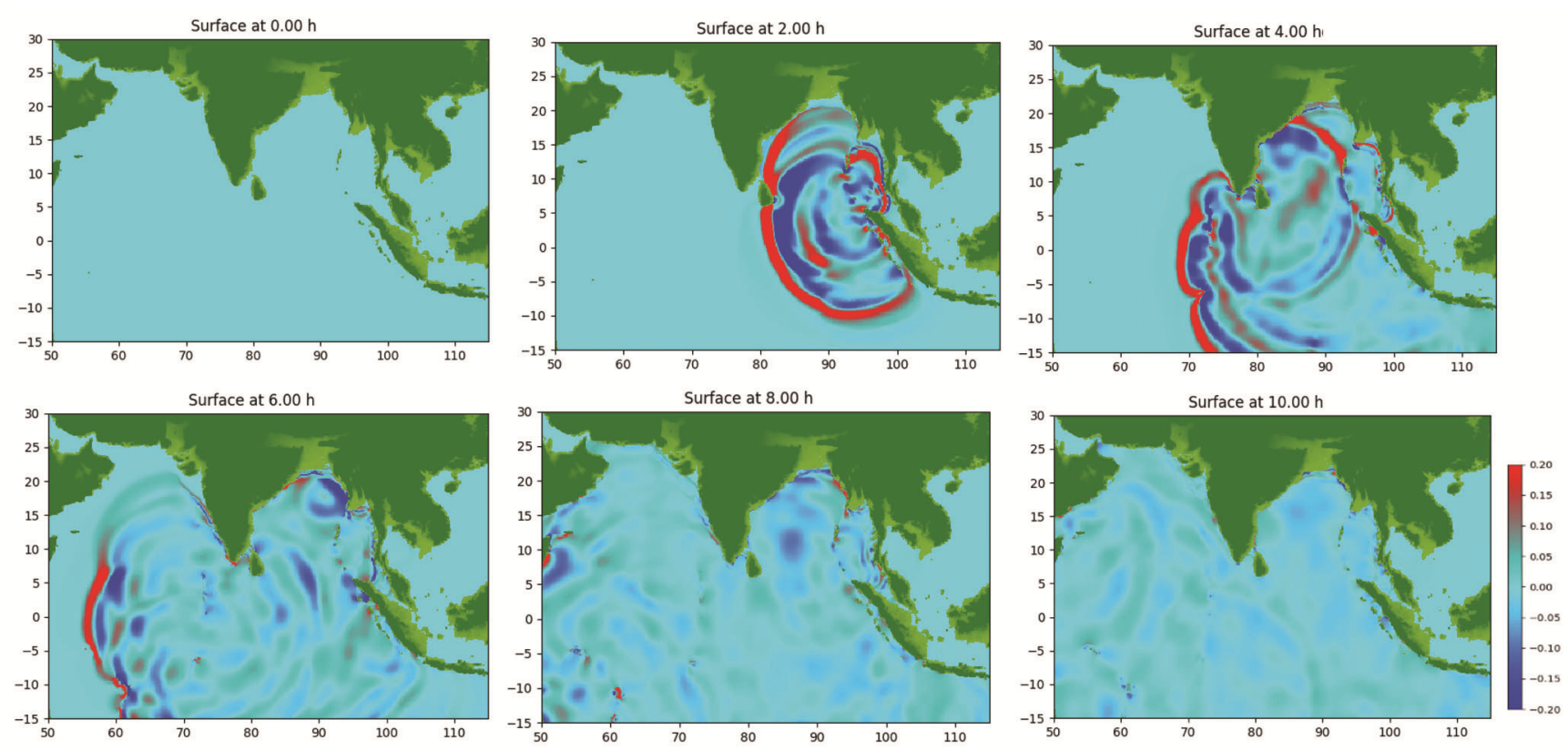

Figure 5. Pattern of propagation of simulated tsunami waves (m) at different time intervals for the $2004 M_{w} 9.12$ Sumatra earthquake slip field. The nonlinear distribution of the wave due to bathymetry variations and pattern of slip field can be seen from the propagation pattern.

performed to obtain synthetic waves for $15 \mathrm{~h}$ from the initiation of tsunami waves at the source.

\section{Validation}

The next step lies in the evaluation of the performance of the proposed tsunami generation procedure adopted for the study. Hence, the tsunami waves are generated for the $2004 M_{w} 9.12$ Sumatra earthquake. The source characteristics are taken from the models available in the SRCMOD database (Figure 2) with the corresponding displacement field simulated from quasi-static Okada's solution. It should be noted here that each slip field is generated based on different datasets and inversion methodologies. Here, slip fields from researchers ${ }^{25,26}$ utilized the tele-seismic data, while those in ref. 27 considered 10-IRIS station records and GPS-GEOSCOPE seismic station readings. It is also clear that the slip fields vary in dimension, orientation and finiteness. The differences in slip characteristics are reflected in the final displacement field as well. Furthermore, we performed a comparison on the simulated results with the recorded tidal gauge 

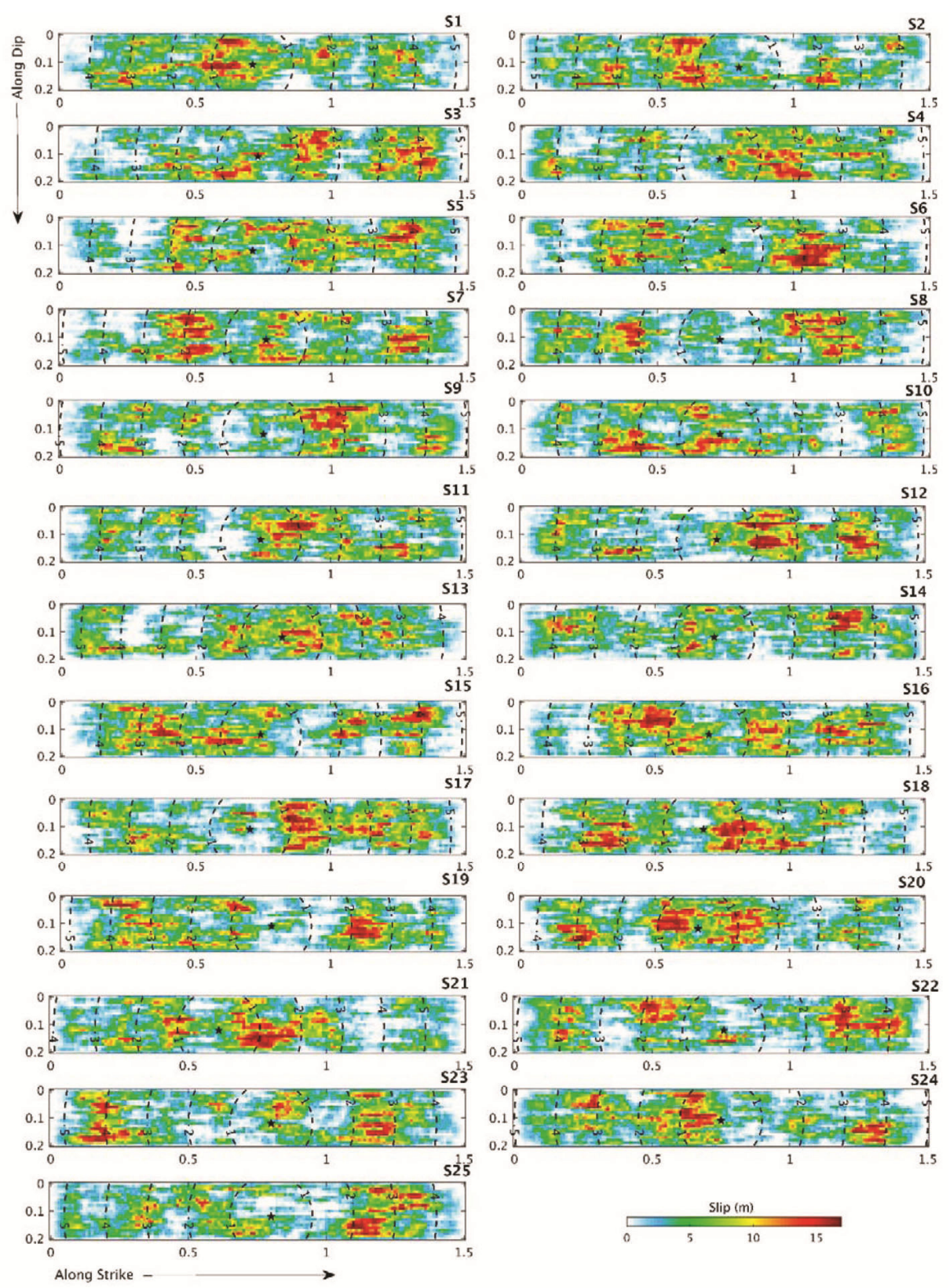

Figure 6. Slip samples corresponding to scenario events with $M_{\mathrm{w}} 9.12$ having truncated exponential probability density and stable variogram.

along the coast and altimeter data through the ocean surface available for the event. The de-tided data are available at Chennai, Paradip, Tuticorin and Vishakhapatnam (http://www.nio.org/index/option/com_nomenu/task/show/ tid/2/si/18/id/11) as described by Nagarajan et al. ${ }^{36}$. Additionally, altimeter data (sea surface elevation) were captured by Jason-1, TOPEX/Poseidon, and ENVISAT satellites as they traversed across the Indian Ocean along the path at 115,120 and 200 min respectively, from the triggering time of the $2004 M_{w} 9.12$ Sumatra earthquake. Figure 4 shows a comparison between the simulated and recorded data. The simulated tsunami waves can capture the pattern, amplitude and phase having significant correlation with the recorded data. However, slight variations between the source models can also be noted from the comparisons. These differences are attributed to the variations associated with the reported slip pattern due to the differences in database and slip field estimation algorithms, as discussed earlier. Figure 5 shows the pattern of wave propagation at different time instants. The nonlinearity in wave propagation pattern due to boundary conditions like bathymetry and slip field is evident from the figure. Thus, the developed numerical model is found to be efficient in modelling the synthetic waves.

Now that the adopted tsunami generation model is reasonably validated, it will be interesting to observe the wave pattern for certain scenario events. The slip distributions for the scenario event could be estimated from 

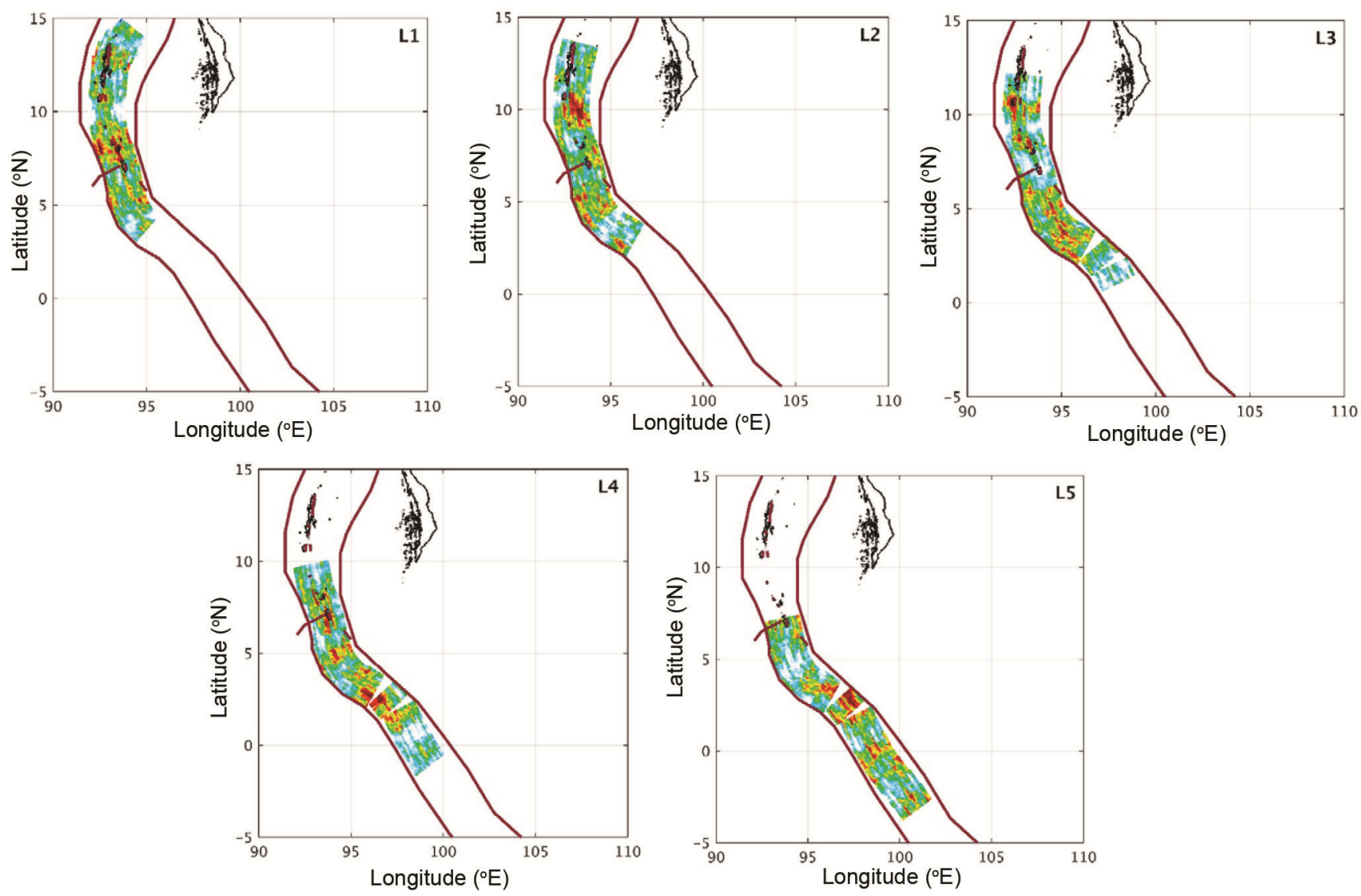

Figure 7. Location and orientation of slip distribution along the Sumatra subduction region considered for performing sensitivity analysis.

the recent methodology proposed for the generation of non-Gaussian random field conserving second-order characteristics in ref. 21. An effort to understand the sensitivity of tsunami waves due to slip distribution and location has been made in the present study. Additionally, for performing probabilistic tsunami hazard analysis, owing to the inherent nonlinearity of the wave, one must resort to numerous simulations for all possible scenario events. Performing such simulations for an ensemble of the source could be computationally expensive. However, the design of a structure along the coastline could be done using an estimate of the maximum possible wave height in the region. Hence as a pilot analysis, the deterministic tsunami hazard along the coastline in the Bay of Bengal region is estimated as detailed further.

\section{Rupture models for the $M_{w} 9.12$ scenario event}

The rupture models for the scenario events are estimated according to the iterative methodology for the generation of non-Gaussian slip ${ }^{21}$. The slip fields are generated for the $M_{w} 9.12$ event which is the maximum magnitude that occurred in the region. The dimension of the rupture model is taken as $1500 \mathrm{~km} \times 195 \mathrm{~km}$ consistent with that observed for the great event in the region, with sub-fault dimension $10 \mathrm{~km} \times 10 \mathrm{~km}$. The probability and variogram structure of the slip fields corresponding to the scenario event are obtained using the scaling relations proposed in ref. 21 for the respective parameters. Thus, the probability structure of the slip distribution is taken as a truncated exponential with $\mu=13.23$ and $D_{\max }=17 \mathrm{~m}$. The theoretical variogram structure is taken as stable with sill $7.16 \times 10^{5}$, range along strike direction $=70 \mathrm{~km}$ and range along down-dip direction $=55 \mathrm{~km}$. In the present study, 25 slip samples have been generated for the region. Figure 6 shows the corresponding fields. It should be noted that the hypocentre location for each of the fields is taken at the maximum probable location following Mai et $a l .{ }^{37}$. The rupture velocity is taken as $2.5 \mathrm{~km} / \mathrm{s}$. Once the slip fields are generated, the next step is to fix the orientation and mechanism of the events. Since pure thrust is the critical mechanism in triggering a tsunami, the rake angle for all the scenario events is considered as $90^{\circ}$. Furthermore, orientation of the fault plane with respect to location, strike and dip is taken considering the coordinates reported by NOAA (https://nctr.pmel.noaa.gov/propagation-database.html) for the Sumatran subduction region. Here strike angle varies from $0.63^{\circ}$ to $353.15^{\circ}$ and dip angle from $7^{\circ}$ to $58^{\circ}$. Here orientations are prescribed for a fault plain of dimension $100 \mathrm{~km} \times 50 \mathrm{~km}$. The generated slip samples from the study are assigned 

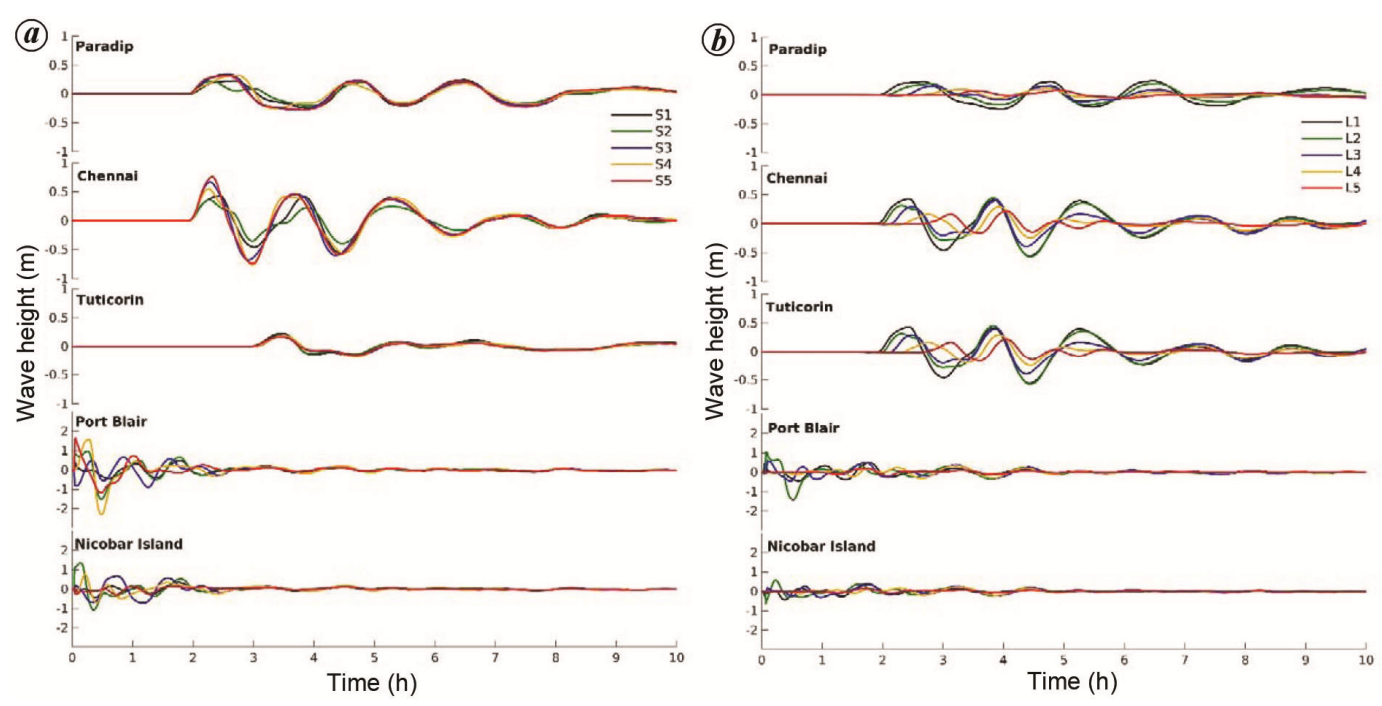

Figure 8. Sensitivity analysis with respect to finiteness of slip field and location of slip distribution. (a) Same location (L1), different slip fields (S1-5) and (b) Different locations (L1-5), same slip field (S1).

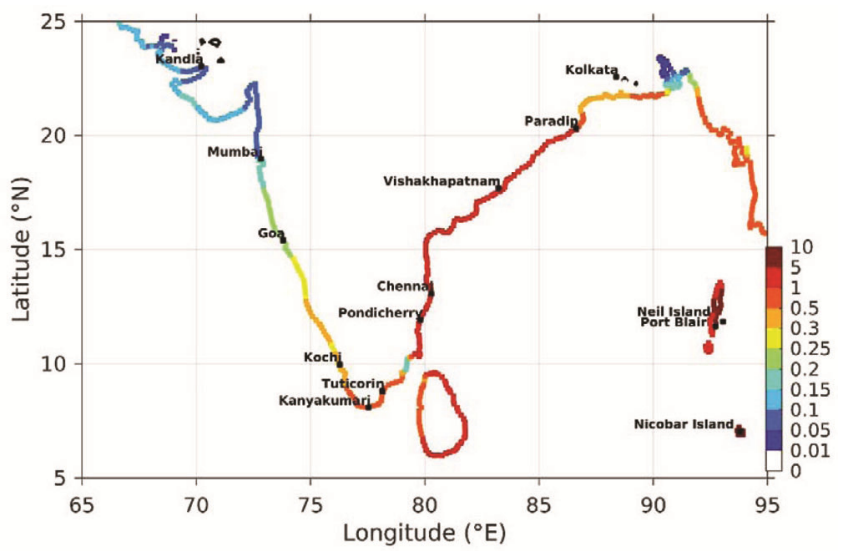

Figure 9. Deterministic tsunami hazard with respect to maximum wave height $(\mathrm{m})$ along the coastline of the study region with respect to maximum values from all scenario events.

with proper orientation and mechanism according to the region at five sets of locations (Figure 7).

\section{Sensitivity analysis}

Now, it is important to understand how the slip distribution and location of the rupture plane will affect the tsunami waves that are generated. Here wave height at two near-field sites (Port Blair $-11.63^{\circ} \mathrm{N}, 92.72^{\circ} \mathrm{E}$ and Nicobar Island $-7.03^{\circ} \mathrm{N}, 93.80^{\circ} \mathrm{E}$ ) and three far-field sites (Paradip $-20.32^{\circ} \mathrm{N}, 86.62^{\circ} \mathrm{E}$, Chennai $-13.10^{\circ} \mathrm{N}$, $80.30^{\circ} \mathrm{E}$ and Tuticorin $-8.81^{\circ} \mathrm{N}, 78.14^{\circ} \mathrm{E}$ ) are considered for comparison. First the effect of slip distribution on tsunami waves is assessed by estimating the waveform using slip fields S1 to S5 (Figure 6) at location 1 (Figure 7). A uniform rupture velocity of $2.5 \mathrm{~km} / \mathrm{s}$ is considered for all rupture models. Accordingly, it will take almost $315 \mathrm{sec}$ to rupture the full plane considering the hypocentre location. Figure $8 a$ shows the corresponding waveforms at five selected stations. It is noted that at near-field stations, the variations with respect to slip fields are high. While at far-field stations, the observed corresponding variability is less. Furthermore, to understand the effect of location on tsunami waveforms, slip field corresponding to S1 (Figure 6) is applied at L1-L5 (Figure 7). Figure $8 b$ shows the corresponding waveforms at the selected sites. The wave amplitude is observed to decrease as the location shifts from L1 to L5. Further, the variability is observed for both near and far-field sites. The major observations from the sensitivity study performed here are that the near-field stations are affected by both variations in slip field as well as slip location. However, far-field stations are more sensitive towards the location of rupture rather than finiteness of the fault.

\section{Deterministic hazard for maximum wave height along the coast}

Next, it will be interesting to estimate deterministic tsunami hazards with respect to maximum wave heights in the coastal areas of South Asia due to earthquakes along the Sumatran region. Both finiteness in fault and rupture locations are considered while estimating the hazard. Accordingly, the results are based on 25 simulations corresponding to the synthetic slip fields generated for $M_{w} 9.12$. It should be noted that $M_{w} 9.12$ is the extreme event that occurred in the region. Hence, it can be considered as the worst-case scenario for estimation of deterministic tsunami hazard. However, the event had ruptured only a certain portion of the subduction region, and there is possibility of occurrence of events of similar scale as and when enough strain gets accumulated due to active tectonics. Additionally, as discussed earlier in the text, the wave heights at a site are sensitive to finiteness of the rupture and its location. Hence it is important to ascertain the worst possible wave height along the study region. 

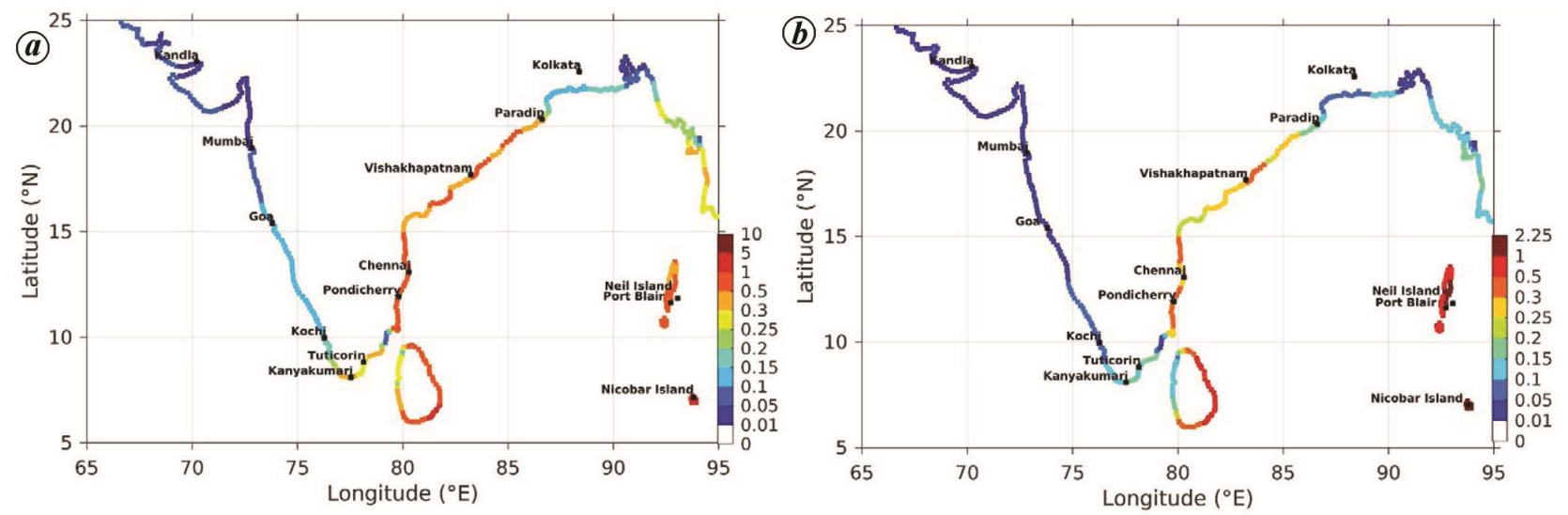

Figure 10. Deterministic tsunami hazard with respect to maximum wave height $(\mathrm{m})$ along the coastline of the study region with respect to mean and standard deviation of maximum wave heights from all scenario events. $\boldsymbol{a}$, Mean of maximum wave height; $\boldsymbol{b}$, Standard deviation of maximum wave height.

For this, a set of samples is taken along the possible rupture zone inclusive of rupture finiteness and possible location variability. Thus, simulations are performed with $\mathrm{S} 1-\mathrm{S} 5$ at L1, S6-S10 at L2, S11-S15 at L3, S16-S20 at L4 and S21-S25 at L5 (Figures 6 and 7). Further, wave height along the coast where the initial mean sea level is zero is extracted after tsunami wave simulations. Figure 9 shows the possible maximum wave height along the coast for all the cases using the simulated data. The maximum wave height of the order $10 \mathrm{~m}$ is observed at the Andaman \& Nicobar region. Along the east coast of India, the value ranges from 1 to $7 \mathrm{~m}$. The mean of the maximum wave heights for all cases, along with the standard deviation, is shown in Figure $10 a$ and $b$ respectively. The wave amplitude is observed in the range 2 to $5 \mathrm{~m}$. The presence of Sri Lankan land mass acts as a shield for the southern portion of India's coast from the direct impact of tsunami waves. Relatively less wave height at the Kolkata region is attributed to the directivity of wave propagation due to the orientation of faults in the Sumatran region. Along the west coast, the maximum wave height of the order of $2-3 \mathrm{~m}$ is observed towards the southern tip. The mean amplitude is observed at around $0.3 \mathrm{~m}$. The standard deviation among waveforms is observed to be maximum along the Andaman region of the order $2.25 \mathrm{~m}$. Along the west coast, the maximum standard deviation of the order $0.5 \mathrm{~m}$ is observed for some parts of Tamil Nadu and Andhra Pradesh. These results give an estimate of the maximum possible wave heights along the coastline of the Bay of Bengal. This information can be considered in the design and construction of huge infrastructural projects like nuclear power plants, ports, etc.

\section{Summary and conclusion}

The present study estimates the deterministic tsunami hazard values corresponding to maximum wave height for the coastline of India and adjoining regions due to subduction events in the Sumatra region. The study accounts for source randomness and location in the computation. Here, computationally efficient quasi-static Okada's solution is used as a substitute to simulate and understand the dynamic ground motion pattern in the near field. The approach is observed to capture the trend in the displacement as well as pattern of ground residual displacement. Hence the quasi-static Okada's solution is utilized to quantify the dynamic ocean-floor displacement field due to slip models in the SRCMOD database for the 2004 Sumatra earthquake. Furthermore, these displacement fields are employed to simulate tsunami waves utilizing Clawpack package, which has coded a standard finite volume framework formulated based on shallow-water wave equation. The resultant simulated waveforms are compared with the available tidal gauge recordings and altimeter data. The simulated waves are observed to capture the trends in the recorded data.

The sensitivity of tsunami waves with slip field and location is also evaluated. It is observed that both location and pattern of the slip field affect the waveforms in the nearfield. However, the predominant factor that affects the wave height at far-field stations is the location of rupture. This study also estimates the deterministic tsunami hazard with respect to maximum wave height. The estimates are arrived from simulations corresponding to the worst earthquake that can occur in the region combining 25 scenario simulations along the possible rupture plane at the Sumatra subduction. The maximum wave height observed for the region is of the order of $10 \mathrm{~m}$.

Results from the study indicate the high sensitivity of tsunami waves on input slip distribution. We also noted that the rupture propagation characteristics with respect to slip field and its location have implications on the simulated waves. The results provide a conservative estimate of the maximum wave height along the coast at zero mean sea level. The results can be suitably extrapolated for sites along the shore region based on topography. However, a more scientific method accounting for all the uncertainty would be to perform probabilistic 
tsunami hazard assessment. However, the computational cost is huge as all possible events, corresponding randomness and location need to be accounted and hence will be attempted as a future scope of this work. Additionally, sensitivity analysis is performed for the distribution pattern and location of the rupture model. More insight into the controlling parameters could be gained if all parameters associated with the rupture process are considered for sensitivity analysis. Nevertheless, the results from this study can be utilized in the safe design of structures along the coastline of the study region.

1. Takahasi, R., An estimate of future tsunami damage along the Pacific coast of Japan. Bull. Earthquake Res. Inst., 1951, 29, 71-95.

2. Loomis, H., Tsunami wave runup heights in Hawaii, Technical report, MG-76-5, Hawaii Institute of Geophysics, University of Hawaii, 1976.

3. Houston, J. R., Carver, R. D. and Markle, D. G., Tsunami-wave elevation frequency of occurrence for the Hawaiian Islands. Technical report, Army Engineer Waterways Experiment Station Vicksburg Miss, 1977.

4. Houston, J. R. and Garcia, A. W., Type 16 flood insurance study: tsunami predictions for Pacific coastal communities. Technical report, Army Engineer Waterways Experiment Station Vicksburg Miss, 1974.

5. Rikitake, T. and Aida, I., Tsunami hazard probability in Japan Bull. Seismol. Soc. Am., 1988, 78(3), 1268-1278.

6. Geist, E. L. and Parsons, T., Probabilistic analysis of tsunami hazards. Nat. Hazards, 2006, 37(3), 277-314.

7. González, F. et al., Probabilistic tsunami hazard assessment at seaside, Oregon, for near-and far-field seismic sources. J. Geophys. Res.: Oceans, 2009, 114(C11); doi:10.1029/2008JC005132.

8. Lorito, S., Selva, J., Basili, R., Romano, F., Tiberti, M. and Piatanesi, A., Probabilistic hazard for seismically induced tsunamis: accuracy and feasibility of inundation maps. Geophys. J. Int., 2014, 200(1), 574-588.

9. Bilham, R., Engdahl, R., Feldl, N. and Satyabala, S., Partial and complete rupture of the Indo-Andaman plate boundary 18472004. Seismol. Res. Lett., 2005, 76(3), 299-311.

10. Ortiz, M. and Bilham, R., Source area and rupture parameters of the 31 December $1881 M_{\mathrm{w}}=7.9 \mathrm{Car}$ Nicobar earthquake estimated from tsunamis recorded in the Bay of Bengal. J. Geophys. Res.: Solid Earth, 2003, 108(B4); doi:10.1029/2002JB001941.

11. Pailoplee, S. and Choowong, M., Earthquake frequencymagnitude distribution and fractal dimension in mainland Southeast Asia. Earth, Planets Space, 2014, 66(1), 8.

12. Murty, T., Nirupama, N., Nistor, I. and Hamdi, S., Far field characteristics of the tsunamiof 26 December 2004. ISET J. Earthquake Technol., 2005, 42(4), 213-217.

13. Singh, A., Murty, T., Rastogi, B. and Yadav, R., Earthquake generated tsunami in the Indian ocean and probable vulnerability assessment for the east coast of India. Mar. Geodesy, 2012, 35(1), 49-65.

14. Roshan, A., Basu, P. C. and Jangid, R., Tsunami hazard assessment of Indian coast. Nat. Hazards, 2016, 82(2), 733-762.

15. Geist, E. L., Complex earthquake rupture and local tsunamis. J. Geophys. Res.: Solid Earth, 2002, 107(B5); doi:10.1029/ 2000JB000139.

16. Mavroeidis, G. P. and Papageorgiou, A. S., A mathematical representation of near-fault ground motions. Bull. Seismol. Soc. Am., 2003, 93(3), 1099-1131.

17. Davies, G., Horspool, N., and Miller, V., Tsunami inundation from heterogeneous earthquake slip distributions: evaluation of synthetic source models. J. Geophy. Res.: Solid Earth, 2015, 120(9), 6431-6451.

18. Dhanya, J. and Raghukanth, S. T. G., Ground motion simulation for earthquakes in Sumatran region. Curr. Sci., 2018, 114(8), 1709-1720.

19. Dhanya, J. and Raghukanth, S. T. G., Implication of source models on tsunami wave simulations for $2004\left(M_{\mathrm{w}}\right.$ 9.2) Sumatra earthquake. Nat. Hazards, 2020, 1-26; doi:10.1007/s11069-020-04168-5.

20. George, D. L., Finite volume methods and adaptive refinement for tsunami propagation and inundation. $\mathrm{Ph} \mathrm{D}$ dissertation, University of Washington, USA, 2006.

21. Dhanya, J. and Raghukanth, S. T. G., A non-Gaussian random field model for earthquake slip. J. Seismol., 2019, 23(4), 889-912.

22. Dutykh, D., Mitsotakis, D., Gardeil, X. and Dias, F., On the use of the finite fault solution for tsunami generation problems. Theor. Comput. Fluid Dyn., 2013, 27(1-2), 177-199.

23. Okada, Y., Surface deformation due to shear and tensile faults in a half-space. Bull. Seismol. Soc. Am., 1985, 75(4), 1135-1154.

24. Somerville, P. et al., Characterizing crustal earthquake slip models for the prediction of strong ground motion. Seismol. Res. Lett., 1999, 70(1), 59-80.

25. Ji, C., Preliminary rupture model for the December 26, 2004 earthquake, off the west coast of northern Sumatra, magnitude 9.1. 2005; http://neic.usgs.gov/neis/eq_depot/2004/eq_041226/neic_ slav_ff.html.

26. Ammon, C. J. et al., Rupture process of the 2004 SumatraAndaman earthquake. Science, 2005, 308(5725), 1133-1139.

27. Rhie, J., Dreger, D., Burgmann, R. and Romanowicz, B., Slip of the 2004 Sumatra-Andaman earthquake from joint inversion of long-period global seismic waveforms and GPS static offsets. Bull. Seismol. Soc. Am., 2007, 97(1A), S115-S127.

28. Whitham, G. B., Linear and Nonlinear Waves, John Wiley, New York, 1999.

29. Titov, V. V. and Gonzalez, F. I., Implementation and testing of the method of splitting tsunami (MOST) model. NOAA Technical Memorandum ERL PMEL-112, 1997, pp. 1-11.

30. Imamura, F., Review of tsunami simulation with a finite difference method. Long-waves runup models, 1996, pp. 25-42.

31. Imamura, F., Yalciner, A. C. and Ozyurt, G., Tsunami Modelling Manual. UNESCO IOC International Training Course on Tsunami Numerical Modelling, 2006.

32. LeVeque, R. J., George, D. L. and Berger, M. J., Tsunami modelling with adaptively refined finite volume methods. Acta Numer., 2011, 20, 211-289.

33. George, D., Adaptive finite volume methods with well-balanced Riemann solvers for modeling floods in rugged terrain: application to the Malpasset dam-break flood (France, 1959). Int. J. Numer. Meth. Fluids, 2011, 66(8), 1000-1018.

34. Isvoranu, D. and Badescu, V., Hydrodynamics of tsunamis generated by asteroid impact in the black sea. Central Eur. J. Phys., 2012, 10(2), 429-446.

35. Turmel, D., Locat, J., Leblanc, J. and Cauchon-Voyer, G., Tsunami modelling of the $7250 \mathrm{cal}$ years BP Betsiamites submarine landslide. Geol. Soc., London, Spec. Publ., 2018, 477(1), 293-301.

36. Nagarajan, B. et al., The great tsunami of 26 December 2004: a description based on tide-Gaugedata from the Indian subcontinent and surrounding areas. Earth, Planets Space, 2006, 58(2), 211215.

37. Mai, P. M., Spudich, P. and Boatwright, J., Hypocenter locations in finite-source rupture models. Bull. Seismol. Soc. Am., 2005, 95(3), 965.

Received 10 March 2020; revised accepted 19 August 2020

doi: $10.18520 / \mathrm{cs} / \mathrm{v} 119 / \mathrm{i} 10 / 1641-1651$ 